\title{
Problems of Creation and Design of Engineering Systems: Mathematics, Technology and Informatics
}

\author{
Mini Review \\ Volume 1 Issue 1- 2020
}

\begin{abstract}
Author Details
Mashunin Yu K*

Professor, Far Eastern Federal University, Vladivostok, Russia

*Corresponding author

Mashunin Yu K, Professor, Far Eastern Federal University, Vladivostok, Russia

Article History

Received: December 12, 2020 Accepted: December 18, 2020 Published: December 21, 2020
\end{abstract}

\begin{abstract}
In this work, we presented the problems that arise when automating the creation and design of engineering systems. These problems include: first, mathematical problems that are associated with a variety of functional characteristics of engineering systems, secondly, technological issues, which include the development of technical specifications, the construction of a mathematical and numerical model of an engineering system, taking into account the set of functional characteristics and constraints of the system, and, thirdly, information problems associated with solving problems in conditions of uncertainty (regression analysis) , creation of adequate software. To solve the presented problems, we presented a presentation of two works. In the field of mathematics, the monograph "Theory and methods of vector optimization" is presented, which is a development of the work https://rdcu.be/bhZ8i. In the field of technological and information problems, the article "Mathematical apparatus for selection of optimal parameters of Technical, Technological Systems and Materials based on Vector Optimization ".
\end{abstract}

Keywords: Vector optimization, Methods for solving vector problems; Engineering system modeling

\section{Problems of choosing the optimal parameters of engineering systems in conditions of certainty and uncertainty based on vector optimization}

As an object of research, we consider "Engineering systems", which in General can include technical systems, technological processes, and materials. When researching, analyzing and designing engineering systems, the problem arises of choosing the optimal parameters of engineering systems according to their functional characteristics. Design and implementation of engineering systems include the following stages:

Selection of the best technical parameters and characteristics;

Computer-aided design (CAD);

\section{Experimental and industrial operation}

Currently, the stage of selecting the best parameters of engineering systems is the least formalized stage and is usually solved using expert assessments, as well as "trial and error". The practical part of the work is aimed at automating this stage. In $[1,2]$ the concept is presented as a system of views on the problem of design automation. The study of the engineering system was performed, first, under conditions of certainty, when data on the functional characteristics of the engineering system are known; secondly, under conditions of uncertainty, when the discrete (experimental) values of individual characteristics are known; data on the restrictions that are imposed on the functioning of the system are also known. In organizational terms, the concept of modeling engineering systems in terms of certainty and uncertainty is presented in the form of a flowchart in figure 1. A detailed description of each block in General is provided in [1].

The methodology for selecting the optimal parameters of engineering systems is based on three practical problems: the technical system, the technological process, and the choice of the optimal structure of the material. The methodology includes a number of stages.

a. Formation of the technical specification (source data) for numerical modeling and choice of optimum parameters of a system. The designer who projects the system forms the initial data.

b. Construction of mathematical and numerical models of the technical system in terms of certainty and uncertainty.

c. The solution of the vector problem of mathematical programming (VPMP) - a model of the Engineering system at equivalent criteria.

d. Creation of geometrical interpretation of results of the decision in a three-dimensional coordinate system in relative units. 
e. The solution of a vector problem of mathematical programming - a model of the Engineering system at the given priority of the criterion.

f. Geometrical interpretation of results of the decision in a threedimensional coordinate system in physical units

To implement the presented methodology, it is necessary to solve three major problems.

i. Mathematics. Mathematical methods must solve multi-criteria or vector problems of mathematical programming. A presentation of solutions to such problems is presented in the next section.

ii. Technological problems, which include the formation of a technical specifications, the construction of a mathematical and numerical model of an engineering system, which depend on a set of functional characteristics of the system. The presentation of the solution to such problems is presented in the third section, [3-11, and 13].

iii. Information problems associated with solving problems in conditions of uncertainty (regression analysis), the creation of adequate software - presented in the fourth section.

\section{Mathematics}

Vector problem of mathematical programming. Presentation of the monograph "Theory and Methods of Vector Optimization"

A vector problem in mathematical programming (VPMP) is a standard mathematical-programming problem including a set of criteria, which, in total, represent a vector of criteria

It is important to distinguish between uniform and non-uniform VPMP:

A uniform maximizing VPMP is a vector problem in which each criterion is directed towards maximizing;

A uniform minimizing VPMP is a vector problem in which each criterion is directed towards minimizing;

A non-uniform VPMP is a vector problem in which the set of criteria is shared between two subsets (vectors) of criteria (maximization and minimization respectively), e.g., non-uniform VPMP are associated with two types of uniform problems.

According to these definitions, we will present a vector problem in mathematical programming with non-uniform criteria $[14,15]$ in the following form:

$$
\begin{aligned}
\text { Opt } F(x)= & \max F_{1}(X)=\left\{\max f_{k}(X), \overline{k=1, K_{1}}\right. \\
& \left.\min F_{2}(X)=\left\{\min f_{k}(X), \overline{k=1, k_{2}}\right\}\right\}
\end{aligned}
$$$$
G(X) \leq B \text {, }
$$$$
X \geq 0 \text {, }
$$

Where $X=\left\{x_{j}, j=\overline{1, N}\right\}$ is a vector of material variables, $N$-dimensional Euclidean space of $R^{N}$, (designation $j=j=\overline{1, N}$ \} is eauivalent to $j=1, \ldots, N)$;

$F(X)$ is a vector function (vector criterion) having $K$-a component functions, $(K$ - set power $K), \quad F(X)=\left\{f_{k}(X), k=\overline{1, K_{1}}\right\}$. The set $K$ consists of sets of $K_{1}$, a component of maximization and $K_{2}$ of minimization; $\boldsymbol{K}=\boldsymbol{K}_{1} \cup \boldsymbol{K}_{2}$ therefore we enter the designation of the operation "opt," which includes max and min;

$F_{1}(X)=\left\{f_{k}(X), k=\overline{1, K_{1}}\right\}$ is maximizing vector-criterion, $K_{1}$ - number of criteria, and $K_{1} \equiv \overline{1, K_{1}}$ is a set of maximizing criteria (a problem (2.1), (2.3), (2.4) represents VPMP with the homogeneous maximizing criteria). Let's further assume that $f_{k}(X), k=\overline{1, K_{1}}$ is the continuous concave functions (we will sometimes call them the maximizing criteria);
$F_{2}(X)=\left\{f_{k}(X), k=\overline{1, K_{2}}\right\}$ is vector criterion in which each component is minimized, $K_{2} \equiv \overline{K_{1}+1, K} \equiv \overline{1, K_{2}}$ - a set of minimization criteria, $\boldsymbol{K}_{2}$ - number, (the problems (2.2)-(2.4) are VPMP with the homogeneous minimization criteria). We assume that $f_{k}(X), k=\overline{1, K_{2}}$ is the continuous convex functions (we will sometimes call these the minimization criteria), i.e., $\boldsymbol{K}_{1} \cup \boldsymbol{K}_{2}=\boldsymbol{K}, \boldsymbol{K}_{1} \subset \boldsymbol{K}, \boldsymbol{K}_{2} \subset \boldsymbol{K}$.

$G(X) \leq B, X \geq 0$ is standard restrictions, $g_{i}(X) \leq b_{i}, i=1, \ldots, M$ where $b_{i}$ - a set of real numbers, and $g_{i}(X)$ are assumed continuous and convex.

$S=\left\{X \in R^{n} \mid X \geq 0, G(X) \leq B, X^{\min } \leq X \leq X^{\max }\right\} \neq \varnothing$

where the set of admissible points set by restrictions (2.3) -(2.4) is not empty and represents a compact.

The vector minimization function (criterion) $F_{2}(X)$ can be transformed to the vector maximization function (criterion) by the multiplication of each component of $F_{2}(X)$ to minus unit. The vector criterion of $F_{2}(X)$ is injected into VPMP (2.1) -(2.4) to show that, in a problem, there are two subsets of criteria of $\boldsymbol{K}_{1}, \boldsymbol{K}_{2}$ with various directions of optimization.

Presentation of the monograph "Theory and Methods of Vector Optimization"

To solve theoretical questions and develop methods for solving the VZMP (2.1) - (2.4) We present the monograph "Theory and Methods of Vector Optimization" which includes the following chapters.

Chapter 1. Vector problems in Mathematical Programming (VPMP).

Chapter 2. The theoretical bases of vector optimization.

Chapter 3. Methods for solving problems OF vector optimization.

Chapter 4. Research and analysis of approaches to problem-solving in vector optimization.

Chapter 5. The theory of vector problems in mathematical programming with independent criteria.

Chapter 6. The duality of vector problems in linear programming (VPLP).

Chapter 7. the theory of management decision-making based on vector optimization.

\section{Vector problem of mathematical programming with conditions of certainty and uncertainty.}

Presentation of the review «Mathematical apparatus for selection of optimal parameters of Technical, Technological Systems and Materials based on Vector Optimization»

A vector problem of mathematical programming with conditions of certainty and uncertainty. The Mathematical models of a technical system (1a)-(4a), technological process (1b)-(4b) and structures of material (1c)-(4c) in [15] are constructed in the assumption that the functional dependence of each criterion (characteristic) and restrictions from parameters of the studied object is known. In real life such functional dependence of criterion from parameters extremely infrequent, i.e. there are conditions of uncertainty. We present a vector optimization problem with certainty and uncertainty conditions.

The conditions of a certainty are characterized by the fact that the functional dependence of each characteristic and restrictions on the parameters of the studied object is known, $[3,6,8,10]$.

The conditions of uncertainty are characterized by the fact that the initial data characterizing the studied object are presented:
a. random,
b. fuzzy, or, 
c. incomplete data.

Therefore, we lack sufficient information about the functional dependence of each characteristic and restrictions on the parameters $[1,6,11]$.

For options

a) and

b) basic data have to be transformed to option

c) and are presented in the table form.

In work, the option c) - with not full data, which are, as a rule, obtained from experimental data, is investigated.

In real life, the conditions of certainty and uncertainty are combined. The process model should also reflect these conditions. We will present a model of the technological process under certainty and uncertainty taken together:

$$
\begin{gathered}
\text { Opt } F(X)=\left\{\max F_{1}(X)=\left\{\max f_{k}(X), k=\overline{1, K_{1}^{\text {def }}}\right\},\right. \\
\left.\max I_{1}(X)=\left\{\max f_{k}\left(X_{i}, i=\overline{1, M}\right)\right\}^{T}, k=\overline{1, K_{1}^{u n c}}\right\} \\
\min F_{2}(X)=\left\{\max f_{k}(X), k=\overline{1, K_{2}^{d e f}}\right. \\
\left.\left.\min I_{2}(X)=\left\{\max f_{k}\left(X_{i}, i=\overline{1, M}\right)\right\}^{T}, k=\overline{1, K_{1}^{u n c}}\right\}\right\} \\
\text { at restriction } f_{k}^{\min } \leq f_{k}(X) \leq f_{k}^{\max }, k=\overline{1, K} \\
x_{j}^{\min } \leq x_{j} \leq x_{j}^{\max }, j=\overline{1, N}
\end{gathered}
$$

Where $X=\left\{x_{j}, j=\overline{1, N}\right\}$ is the vector of controlled variables (input parameters of the studied object). $\quad F(X)=\left\{F_{1}(X) F_{2}(X) I_{1}(X) I_{2}(X)\right\}$ are a vector criterion, each component of which represents a vector of criteria (output characteristics of the studied object). The magnitude of the characteristic (function) depends on the discrete values of the vector of variables $X . X . F_{1}(X), F_{2}(X)$ are the set of functions max and min, respectively. $I_{1}(X), I_{2}(X)$ are the set of discrete values of the characteristics max and min, respectively. $\overline{1, K_{1}^{d e f}}, 1, K_{2}^{\text {def }}$ (definiteness), $\overline{1, K_{1}^{u n c}}, \overline{1, K_{2}^{u n c}}$ (uncertainty) a set of criteria max and min formed under certainty and uncertainty. $\operatorname{In}(3) f_{k}^{\min } \leq f_{k}(X) \leq f_{k}^{\max }, k=\overline{1, K}$ is a vectorfunction of restrictions imposed on the operation of the technological process, $x_{j}^{\min } \leq x_{j} \leq x_{j}^{\max }, j=1, N$ are parametric restrictions imposed on the studied object.

Presentation of the paper "Mathematical apparatus for selection of optimal parameters of Technical, Technological Systems and Materials based on Vector Optimization".

For the solution of theoretical issues and develop solution methods for solving VPMP (3.1)-(3.4) we presented the paper [15], which includes the following sections:

a. Introduction;

b. Setting the problem of selection of optimal parameters of engineering systems;

c. Problem of vector optimization as mathematical model of a technical system, technological process and structure of material with conditions of certainty and uncertainty;

d. Mathematical modeling apparatus: Theory and Methods of vector optimization;

e. Methodology for selecting optimal parameters of engineering systems under conditions of certainty and uncertainty based on vector optimization.
Information and software problems associated with the solution of vector problems (2.1) - (2.4) and (3.1) - (3.4), which represent mathematical models of engineering systems, and which can be solved both under conditions of certainty and uncertainty (with taking into account regression analysis), are presented in [16-27].

\section{References}

1. Yu K Mashunin (2017) Concept of Technical Systems Optimum Designing (Mathematical and Organizational Statement). International Conference on Industrial Engineering, Applications and Manufacturing, ICIEAM 2017 Proceedings 8076394.

2. Yu K Mashunin (2017) Optimum Designing of the Technical Systems Concept (Numerical Realization). International Conference on Industrial Engineering, Applications and Manufacturing, ICIEAM 2017 Proceedings 8076395. Saint Petersburg. Russia.

3. Yu K Mashunin, VL Levitskii (1996) Methods of Vector Optimization in Analysis and Synthesis of Engineering Systems. Monograph (DVGAEU, Vladivostok, 1996) [in Russian].

4. Yu K Mashunin (2000) "Engineering system modeling on the base of vector problem of nonlinear optimization," Control Applications of Optimization, Preprint of the eleventh IFAC International Workshop. CAO 2000 (Saint_Petersburg, 2000).

5. Yu K Mashunin (1999) Solving composition and decomposition problems of synthesis of complex engineering systems by vector_optimization methods, Comput Syst Sci Int 38: 421.

6. Mashunin K Yu, Mashunin Yu K (2013) Simulation Engineering Systems under Uncertainty and Optimal Descision Making. Journal of Comput Syst Sci Int 52(4): 519-534.

7. Mashunin Yu K (2013) Control Theory. The mathematical apparatus of management of the economy. Logos. Moscow, pp. 448.

8. Mashunin Yu K, Mashunin K Yu (2014) Modeling of technical systems on the basis of vector optimization (1. At equivalent criteria). International Journal of Engineering Sciences \& Research Technology 3(9): 84-96.

9. Mashunin Yu K, Mashunin K Yu (2014) Modeling of technical systems on the basis of vector optimization (2. with a Criterion Priority). International Journal of Engineering Sciences \& Research Technology 3(10): 224-240.

10. Yu K Mashunin, K Yu Mashunin (2015) Simulation and Optimal Decision Making the Design of Technical Systems. American Journal of Modeling and Optimization. 3(3): 56-67.

11. Yu K Mshunin, K Yu Mashunin (2016) Simulation and Optimal Decision Making the Design of Technical Systems (2. The Decision with a Criterion Priority). American Journal of Modeling and Optimization4(2): 51-66.

12. Yu K Mashunin (2017) Vector optimization in the system optimal Decision Making the Design in economic and technical systems. International Journal of Emerging Trends \& Technology in Computer Science 7(1): 42-57.

13. Yu K Mashunin (2019) Methodology of the Choice of Optimum Parameters of Technological Process on Experimental to Data." American Journal of Modeling and Optimization 7(1): 20-28.

14. KM Oluwasegun, OA Ojo, OT Ola, A Birur, J Cuddy et al., (2018) Development of Artificial Neural Network Models for Predicting Weld Output Parameters in Advanced Fusion Welding of a Magnesium Alloy. American Journal of Modeling and Optimization 6(1): 18-34.

15. https://www.scirp.org/journal/ajor.

16. Yu K Mashunin (1986) Methods and Models of Vector Optimization (Nauka, Moscow) [in Russian].

17. Mashunin Yu K (2018) Optimal designing in the interrelation Technical system - Materials (Theory). Mathematical methods in engineering and technology: proceedings of international. Science. Conf.: 12 tons $4 /$ under General Ed. A. A. Bolshakova. -SPb.: Publishing house of Polytechnical Institute p. $40-46$.

18. https://rdcu.be/bhZ8i 
19. Mashunin Yu K (2020) Theory and Methods of Vector Optimization. (Volume One). Cambridge Scholars Publishing. London, pp. 183.

20. A Yu Torgashov, VP Krivosheev, Yu K Mashunin and Ch D Holland (2001) Calculation and multiobjective optimization of static modes of mass_exchange processes by the example of absorption in gas separation, Izv Vyssh Uchebn Zaved, Neft' Gaz 3: 82-86.

21. Yu L Ketkov, A Yu Ketkov, M M Shul'ts (2004) MATLAB 6.x.: Numerical Programming. BKhV_Peterburg, St. Petersburg, pp. 672.

22. R L Keeney, H Raiffa (1981) Decisions with Multiple ObjectivesPreferences and Value Tradeoffs.

23. J Johannes (2010) Vector Optimization: Theory, Applications, and Extensions. Springer Verlag, Berlin, Heidelberg, New York, pp. 510.
24. Q Ansari, Y Jen Chih (2010) Recent Developments in Vector Optimization. Springer, Heidelberg, Dordrecht, London, New York.

25. N Hirotaka, Y Yeboon, Y Min (2009) Sequential Approximate Multiobjective Optimization Using Computational Intelligence. Springer Verlag, Berlin, Heidelberg, pp. 197.

26. R. Shankar (2009) Decision Making in the Manufacturing Environment: Using Graft Theory and Fuzzy Multiple Attribute Decision Making Methods. Springer Verlag.

27. T Cooke, H Lingard, N Blismas (2008) The development and evaluation of a decision support tool for health and safety in construction design, Engineering, Construction and Architectural Management 15(4): 336351. 